\title{
Epidemiology, Diagnosis, and Treatment of Scabies in a Dermatology Office
}

\author{
Kathryn L. Anderson, MD, and Lindsay C. Strowd, MD
}

Background: Scabies is a neglected skin disease, and little is known about current incidence and treatment patterns in the United States. The purpose of this study was to examine demographic data, treatment types, success of treatment, and misdiagnosis rate of scabies in an outpatient dermatology clinic.

Methods: A retrospective chart review of patients diagnosed with scabies within the past 5 years was performed.

Results: A total of 459 charts were identified, with 428 meeting inclusion criteria. Demographic data, diagnostic method, treatment choice, misdiagnosis rate, treatment failure, and itching after scabies are also reported. Children were the largest age group diagnosed with scabies, at $38 \%$. Males (54\%) were diagnosed with scabies more than females. The majority of diagnoses were made by visualizing ova, feces, or mites on light microscopy (58\%). At the time of diagnosis, $45 \%$ of patients had been misdiagnosed by another provider. Topical permethrin was the most common treatment used (69\%), followed by a combination of topical permethrin and oral ivermectin $(23 \%)$, oral ivermectin $(7 \%)$, and other treatments $(1 \%)$.

Conclusion: Our findings suggest that more accurate and faster diagnostic methods are needed to limit unnecessary treatment and expedite appropriate therapy for scabies. (J Am Board Fam Med 2017; 30:78-84.)

Keywords: Child, Dermatology, Diagnostic Errors, Feces, Incidence, Ivermectin, Microscopy, Mites, Outpatients, Permethrin, Pruritus, Retrospective Studies, Scabies, Skin Diseases, Treatment Failure

Scabies is a common pruritic skin infestation caused by the Sarcoptes scabiei mite. ${ }^{1,2}$ The estimated worldwide annual prevalence of scabies is 300 million. $^{3}$ Scabies is a major health concern in many developing countries; in 2009 the World Health Organization declared it a neglected skin disease. ${ }^{4}$

Scabies can be difficult to diagnose for even the most experienced physicians, as examination findings can be subtle and patients may not relate key historic clues of severe pruritus that is worse at night, close contacts who have similar symptoms, and a history of exposure. ${ }^{3}$ Examination findings

This article was externally peer reviewed.

Submitted 9 June 2016; revised 12 September 2016; accepted 19 September 2016.

From the Department of Dermatology, Wake Forest School of Medicine, Winston-Salem, NC.

Funding: none.

Conflict of interest: none declared.

Corresponding author: Kathryn L. Anderson, MD, Department of Dermatology, Wake Forest School of Medicine, Medical Center Blvd, Winston-Salem, NC 27157-1071 (E-mail: Anderson.kathryn.lee@gmail.com). include serpiginous white lines indicative of the mite burrowing; these classically occur in the interdigital web spaces, the areolae of female breasts, or the male genitalia, but they may be found on other body sites. Visualizing burrows or the mite on dermoscopy can assist in the diagnosis ${ }^{5-7}$ (Figure 1). A "gold standard" diagnosis relies on the visualization of ova, feces, or the mites themselves using light microscopy $^{8,9}$ (Figure 2). In cases of diagnostic uncertainty, a skin biopsy may be performed. Visualization of the mite in the stratum corneum can also lead to a diagnosis of scabies. ${ }^{3}$

There are a variety of treatments for scabies. Topical permethrin $5 \%$ is widely used and is the most effective, ${ }^{10}$ but it is associated with resistance, poor patient compliance, and allergic reactions. ${ }^{10-12}$ Oral ivermectin, although not approved by the US Food and Drug Administration for the treatment of scabies, is another treatment option; 2 doses are as effective as a single application of topical permethrin. ${ }^{10,13,14}$ Other treatment options include topical lindane, $5 \%$ precipitated sulfur, 
Figure 1. Scabies under dermoscopy (original magnification, $10 \times$ ). Multiple brown, triangle-shaped heads of Sarcoptes scabiei mites are visualized (arrows).

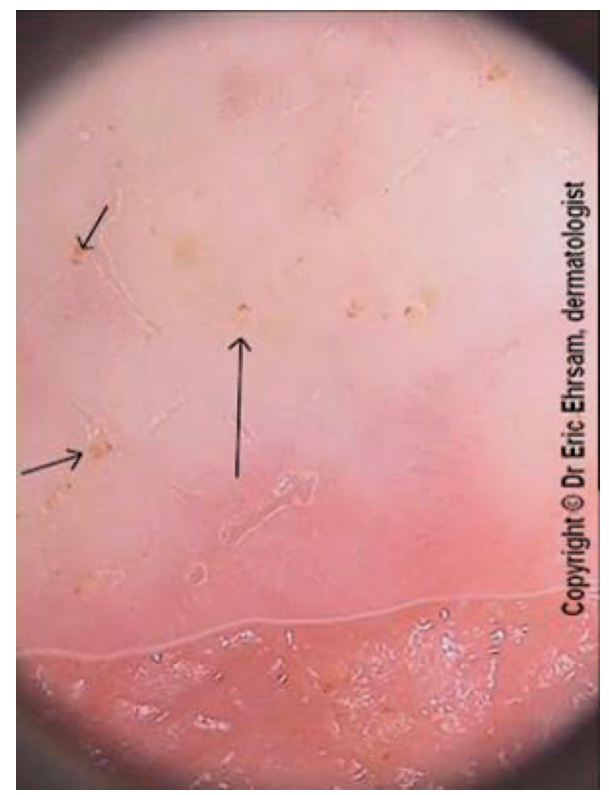

malathion, and topical ivermectin. ${ }^{8,10}$ Treatment choice is limited by $S$ scabiei resistance, cost, availability, and potential toxicity, particularly in children and pregnant women. ${ }^{8,10}$

The high prevalence of scabies in developing countries is attributed to poverty, poor nutritional status, homelessness, and poor hygiene. ${ }^{1}$ In developing countries, the prevalence of scabies is higher among children and adolescents than among adults. ${ }^{1,15,16}$ Scabies affects males and females equally. ${ }^{1}$ In developed countries, scabies outbreaks have historically been described in long-term care facilities, hospitals, and areas of overcrowding. ${ }^{17-20}$ Little is known of the current incidence of and treatment patterns for scabies in the United States. The purpose of this study is to examine demographic data, treatment types, success of treatments, and the misdiagnosis rate of scabies in an outpatient dermatology clinic in the Southeastern United States.

\section{Methods}

After approval by the Wake Forest Baptist Medical Center (WFBMC) Institutional Review Board, the outpatient clinic records at the WFBMC Department of Dermatology were searched for patients who visited the clinic within the past 5 years and had a diagnosis of scabies (International Classification of Diseases, Ninth Revision, code 133.0). Patients were excluded if they were not treated for scabies at the Department of Dermatology.

Patients' age and sex were extracted from the electronic medical record system. Provider notes were reviewed to identify a patient's place of residence, how scabies was diagnosed, the treatment

Figure 2. A Sarcoptes scabiei mite visualized under microscopy (original magnification, 20×) after skin scraping.

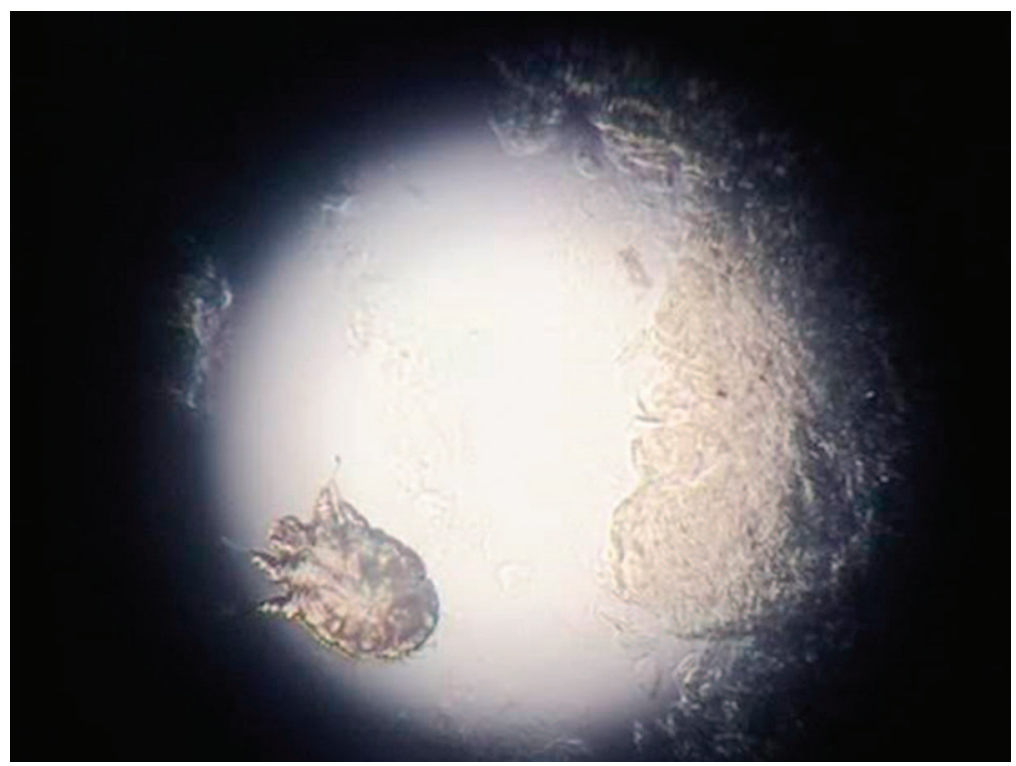


Table 1. Demographic Data of Patients Diagnosed with Scabies at the Wake Forest Baptist Medical Center Department of Dermatology

\begin{tabular}{lc}
\hline $\begin{array}{l}\text { Demographic } \\
\text { Data }\end{array}$ & Patients $(\mathrm{N}=428)$ \\
\hline Age (years) & \\
$0-18$ & $161(38)$ \\
$19-40$ & $88(21)$ \\
$41-65$ & $97(23)$ \\
$>65$ & $82(19)$ \\
Sex & \\
Male & $233(54)$ \\
Female & $195(46)$ \\
Place of residence & \\
Home & $391(91)$ \\
Facility & $30(7)$ \\
Other & $7(2)$ \\
\hline
\end{tabular}

Data are $\mathrm{n}(\%)$.

for scabies, whether the patient was misdiagnosed by another health care provider, the need for retreatment, and the presence of postscabetic itch. If place of residence was not noted in the chart, it was assumed that the patient lives at home. Because ethnicity was not documented in the majority of the charts, it was not included. Descriptive statistics were used to analyze the data. Microsoft Excel (Microsoft Corp, Redmond, WA) was used for data management.

\section{Results}

Based on the diagnosis code and a visit to WFBMC Department of Dermatology, 459 charts were analyzed. A total of 31 charts did not meet inclusion criteria because either no treatment for scabies was received at the WFBMC Department of Dermatology or the chart was miscoded. The remaining
428 charts met inclusion criteria. Demographic information was recorded (Table 1, Figure 3).

Through the providers' notes, the method of diagnosis (Figure 4) and treatment prescribed (Figure 5) were recorded. At the time of scabies treatment by the WFBMC Department of Dermatology, 194 patients $(45 \%)$ had been misdiagnosed and 100 patients $(23 \%)$ had been treated for scabies before presenting to the WFBMC Department of Dermatology. Of the 428 patients treated, $76 \mathrm{pa}-$ tients $(18 \%)$ required additional treatment for scabies at a follow-up appointment (Table 2) and 144 patients $(34 \%)$ had postscabetic dermatitis.

\section{Discussion}

The current literature suggests that scabies is more common among young children compared to adults. ${ }^{1}$ Based on our chart review, the age range of patients diagnosed with scabies was 5 weeks to 92 years, with a median age of 27 years. The pediatric age group ( 0 to 18 years old) had the most scabies diagnoses. There was a slight preponderance of scabies among males compared with females $(54 \%$ vs $46 \%)$. This contradicts the findings of Pannell et $\mathrm{al}^{21}$ who found scabies to be more common in females than males in a long-term retrospective chart review.

Although the literature studying scabies in developed countries describes outbreaks occurring at long-term care facilities and hospitals, ${ }^{17-20}$ the vast majority of patients diagnosed with scabies in this study lived at home. The patients who did not live at home lived at long-term care facilities or in college dormitories.

Health care providers used several different methods to diagnose scabies in this study, and $58 \%$ of the diagnoses were made by seeing the mite or its

Figure 3. Age range and quartiles of patients diagnosed with scabies at Wake Forest Baptist Medical Center.

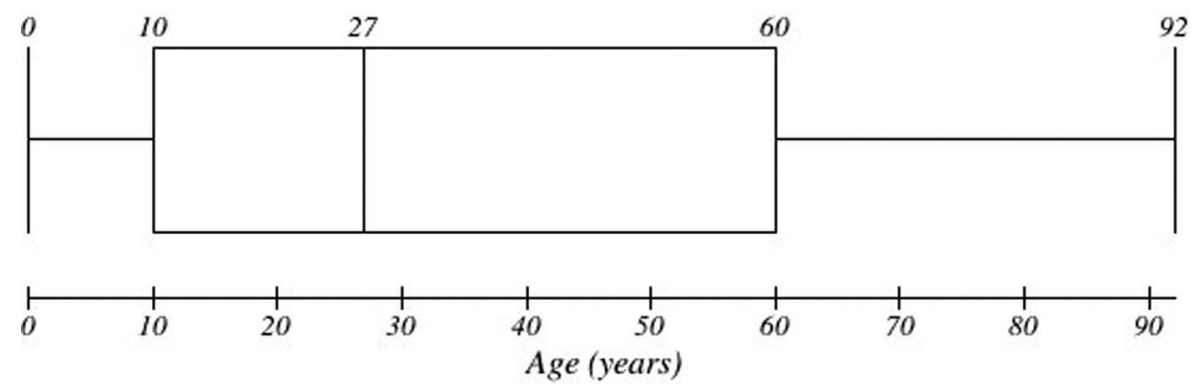


Figure 4. Methods used by providers to diagnose scabies. *Diagnosis via dermoscopy was included in the physical examination group.

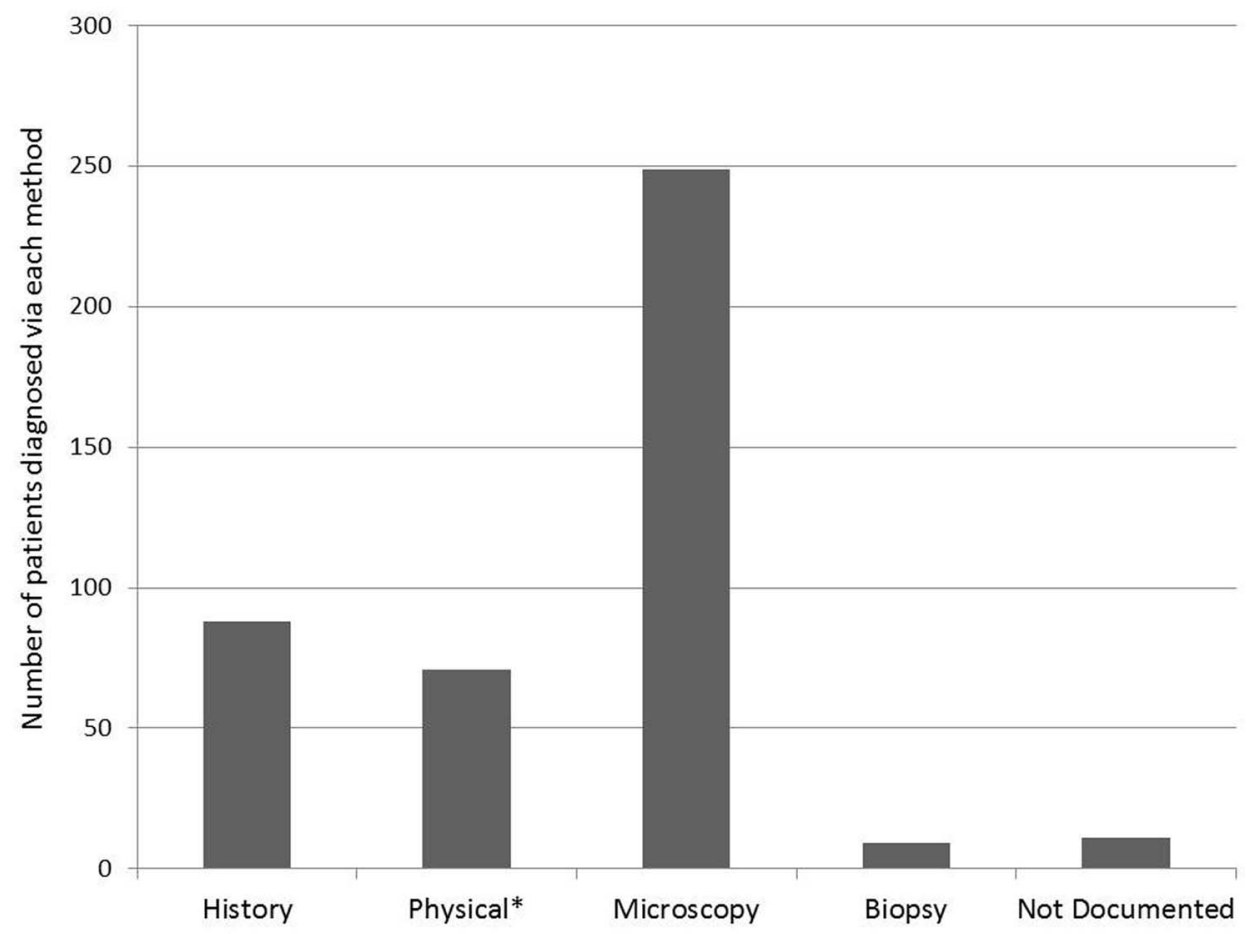

ova or feces upon microscopic examination of a skin scraping. Following microscopy, history and physical examination were the second and third most common methods for diagnosing scabies, respectively. Diagnosis via physical examination included diagnosis via dermoscopy, a highly sensitive method for diagnosing scabies. ${ }^{5}$ Only $2 \%$ were diagnosed via a skin biopsy.

Of the patients diagnosed with scabies, $45 \%$ had been misdiagnosed by another health care provider.
According to old notes in the patients' records, patients were initially misdiagnosed with eczema, papular dermatitis, irritant dermatitis, or contact dermatitis, and 1 patient was misdiagnosed with cutaneous T-cell lymphoma. The misdiagnoses were made by primary care providers, urgent care and emergency department providers, and other dermatologists both within the WFBMC Department of Dermatology and outside it. Awareness of "nonclassic" scabies presentations should be in-

\section{Figure 5. Scabies treatments.}

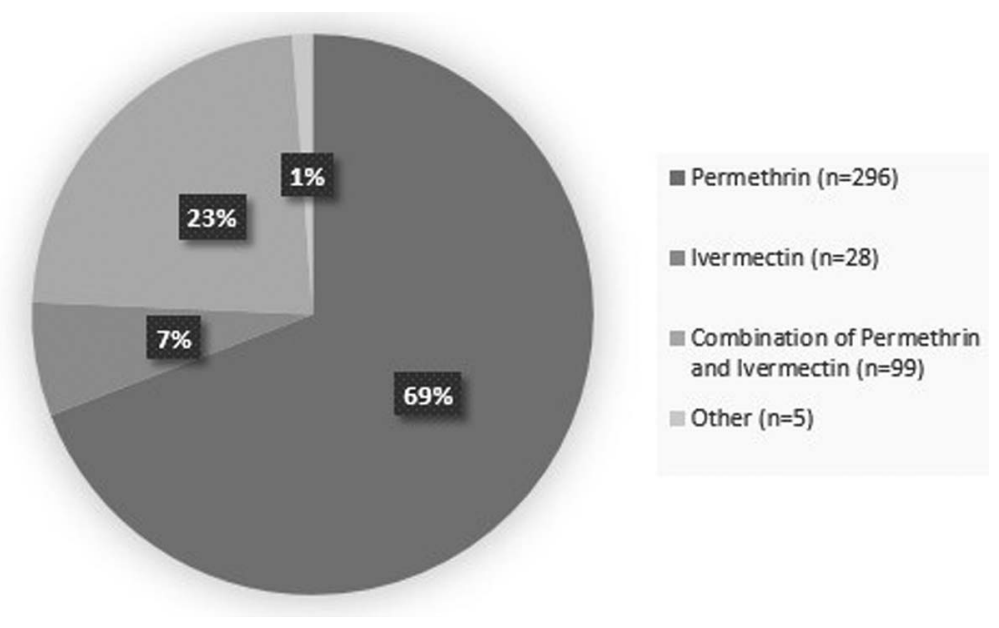


Table 2. Age and Initial Treatment of Patients Who Required Retreatment for Scabies at the Wake Forest Baptist Medical Center Department of Dermatology

Patients $(\mathrm{N}=76)$

\begin{tabular}{lc} 
Age (years) & \\
$0-18$ & $31(41)$ \\
$19-40$ & $10(13)$ \\
$41-65$ & $21(28)$ \\
$>65$ & $14(18)$ \\
Initial treatment & \\
Permethrin & $52(68)$ \\
Ivermectin & $1(1)$ \\
Combination & $21(28)$ \\
Other & $2(3)$ \\
\hline
\end{tabular}

Data are n (\%).

creased among primary care providers, emergency providers, and dermatologists. Patients with severe itching should be questioned not only about their place of residence but also about recent travel history, exposure to day care, and exposure to hospitals. In young children, scabies may present in an unusual distribution involving the face, scalp, and neck, with nodular or pustular lesions as opposed to traditional burrows. ${ }^{8}$ Misdiagnosis exposes patients to potential side effects of unneeded treatment, including the costs of unnecessary treatment. ${ }^{20}$ Two patients in our study received cyclosporine before receiving the correct diagnosis. The frequency of misdiagnosis of scabies supports the need for a more definitive diagnosis option. The use of dermoscopy to diagnose scabies can decrease the false-negative diagnosis rate when compared with skin scrapings. ${ }^{5}$ A serologic test specific for $S$ scabiei has been developed, with $100 \%$ sensitivity and $93.75 \%$ specificity, and may lead to more accurate diagnosis if widely used. ${ }^{22}$

The most common treatment used was topical permethrin as a single agent $(69 \%)$. Although oral ivermectin is not approved by the US Food and Drug Administration for the treatment of scabies, $30 \%$ of patients received oral ivermectin either alone $(7 \%)$ or in combination with permethrin (23\%). Only $1 \%$ of patients were treated with other treatments; in infants this was most often topical precipitated sulfur because the potential adverse effects of topical permethrin in that population. ${ }^{3}$ Resistance to both oral ivermectin and topical permethrin has been described. ${ }^{12}$
Of our study patients, $23 \%$ had received previous treatment for scabies before their initial evaluation at WFBMC; $18 \%$ of study patients required retreatment by WFBMC. The majority of these patients $(68 \%)$ had been treated with permethrin alone; however, over a quarter of them had been treated with a combination of both permethrin and ivermectin. Only 1 patient who had been treated with oral ivermectin alone required retreatment.

There are many possible reasons for treatment failure. If all close contacts are not treated simultaneously, patients may become reinfected, justifying treatment for all close contacts regardless of whether symptoms are present. ${ }^{3,10}$ Many states have legalized Expedited Partner Therapy for sexually transmitted diseases, which would allow prescriptions to be written for contacts of patients with scabies, if scabies is considered a sexually transmitted disease in a particular state. ${ }^{23}$ Patients should also decontaminate all bedding, towels, and clothing at the time of treatment. ${ }^{3,10}$

Nonadherence to the prescribed regimen is another common cause of treatment failure. ${ }^{3,11}$ The instructions for the use of topical permethrin include application of the cream from the neck down in children and adults and over the entire body, including the head, in infants. The permethrin needs to stay on the skin for 8 hours and then be rinsed off, followed by a second application 1 week later. The pediatric age group required the most retreatment, which could be explained by incomplete application or difficulty in applying topical medications on younger patients. Although permethrin is scabicidal, the repeat application is to cover for insufficient application during the first treatment. Ivermectin is also prescribed for 2 treatment courses, but unlike topical permethrin, the reason for 2 courses of ivermectin is because it is scabistatic; the second treatment is meant to kill mites that have hatched since the first treatment. Treatment failure may also be due to $S$ scabiei resistance to topical permethrin and oral ivermectin. ${ }^{12,24-26}$

A common sequela of scabies infestation is persistent itching. This has been attributed to treatment failure, cutaneous irritation, and misdiagnosis. ${ }^{1,3}$ In our study, $34 \%$ of patients (excluding those who required retreatment) had postscabetic complaints. These complaints were attributed to postscabetic itching, Id reaction (autoeczematization), or dermatitic changes caused by irritation from topical medications. Permethrin cream con- 
tains potential allergens, including the permethrin itself, formaldehyde, and other components of the cream base, which can lead to contact dermatitis. ${ }^{14}$ Hypersensitivity reactions after scabies treatment with oral ivermectin are due to the mass release of antigens caused by destruction of the mites and have been described as a reason for transient itching following treatment. ${ }^{14}$ Some cases of continued itching may be due to misdiagnosis; in this study, $40 \%$ of cases were diagnosed without definitive findings of the scabies mite on either light microscopy or biopsy.

This nature of this retrospective chart review has limitations. The study was performed at a single outpatient dermatology site, so the findings may not be generalizable to other settings or other geographic locations. Misdiagnosis rates may be overestimated, as this was a referral center for community primary care and dermatologists. Patients requiring retreatment may be underestimated, as patients may have been subsequently evaluated and treated at other facilities after the initial diagnosis. The retrospective design of the study does not allow for comparisons of diagnostic modalities.

\section{Conclusions}

Scabies is a common skin condition seen in outpatient clinics in developed countries. Patients often present after being misdiagnosed by other providers. Increased awareness of different presentations of scabies, such as signs and symptoms in patients who live at home (rather than in long-term care facilities or those who are homeless), nodular or pustular lesions, and different distributions in children may lead to more accurate and faster diagnosis. The classic method of diagnosing scabies via skin scraping often misses the correct diagnosis because of sampling error. Low-cost, noninvasive diagnostic techniques, such as dermoscopy, may be used during the physical examination. Our findings suggest that more accurate and faster diagnostic methods are needed to diagnose scabies in order to limit unnecessary treatment and expedite appropriate therapy.

The authors thank Eric Ehrsam, MD, for allowing the use of the dermatoscopic image of $S$ scabiei mites.

To see this article online, please go to: http://jabfm.org/content/ 30/1/78. full.

\section{References}

1. Hengge UR, Currie BJ, Jager G, Lupi O, Schwartz RA. Scabies: a ubiquitous neglected skin disease. Lancet Infect Dis 2006;6:769-79.

2. Epidemiology and management of common skin diseases in children in developing countries. Geneva, Switzerland: World Health Organization; 2005.

3. Chosidow O. Clinical practices. Scabies. N Engl J Med 2006;354:1718-27.

4. Feldmeier H, Heukelbach J. Epidermal parasitic skin diseases: a neglected category of poverty-associated plagues. Bull World Health Organ 2009;87:152-9.

5. Dupuy A, Dehen L, Bourrat E, et al. Accuracy of standard dermoscopy for diagnosing scabies. J Am Acad Dermatol 2007;56:53-62.

6. Argenziano G, Fabbrocini G, Delfino M. Epiluminescence microscopy. A new approach to in vivo detection of Sarcoptes scabiei. Arch Dermatol 1997; 133:751-3.

7. Brunetti B, Vitiello A, Delfino S, Sammarco E. Findings in vivo of Sarcoptes scabiei with incident light microscopy. Eur J Dermatol 1998;8:266-7.

8. Johnston G, Sladden M. Scabies: diagnosis and treatment. BMJ 2005;331:619-22.

9. Hicks MI, Elston DM. Scabies. Dermatol Ther 2009;22:279-92.

10. Strong M, Johnstone P. Interventions for treating scabies. Cochrane Database Syst Rev 2007;(3): CD000320.

11. Heukelbach J, Feldmeier H. Scabies. Lancet 2006; 367:1767-74.

12. Thomas J, Peterson GM, Walton SF, Carson CF, Naunton M, Baby KE. Scabies: an ancient global disease with a need for new therapies. BMC Infect Dis 2015;15:250.

13. Goldust M, Rezaee E, Hemayat S. Treatment of scabies: comparison of permethrin $5 \%$ versus ivermectin. J Dermatol 2012;39:545-7.

14. Usha V, Gopalakrishnan Nair TV. A comparative study of oral ivermectin and topical permethrin cream in the treatment of scabies. J Am Acad Dermatol 2000;42:236-40.

15. Hay RJ, Steer AC, Engelman D, Walton S. Scabies in the developing world-its prevalence, complications, and management. Clin Microbiol Infect 2012; 18:313-23.

16. Burkhart CG, Burkhart CN, Burkhart KM. An epidemiologic and therapeutic reassessment of scabies. Cutis 2000;65:233-40.

17. Bouvresse S, Chosidow O. Scabies in healthcare settings. Curr Opin Infect Dis 2010;23:111-8.

18. Ladbury G, Morroy G, van Hoeven-Dekkers S, et al. An outbreak of scabies in multiple linked healthcare settings in The Netherlands. Infect Control Hosp Epidemiol 2012;33:1047-50.

19. Hewitt KA, Nalabanda A, Cassell JA. Scabies outbreaks in residential care homes: factors associated 
with late recognition, burden and impact. A mixed methods study in England. Epidemiol Infect 2015; 143:1542-51.

20. de Beer G, Miller MA, Tremblay L, Monette J. An outbreak of scabies in a long-term care facility: the role of misdiagnosis and the costs associated with control. Infect Control Hosp Epidemiol 2006;27:517-8.

21. Pannell RS, Fleming DM, Cross KW. The incidence of molluscum contagiosum, scabies and lichen planus. Epidemiol Infect 2005;133:985-91.

22. Jayaraj R, Hales B, Viberg L, et al. A diagnostic test for scabies: IgE specificity for a recombinant allergen of Sarcoptes scabiei. Diagn Microbiol Infect Dis 2011;71:403-7.

23. Centers for Disease Control and Prevention. Expedited partner therapy in management of sexually transmitted diseases. Atlanta: US Department of Health and Human Services; 2006.

24. Currie BJ, Harumal P, McKinnon M, Walton SF. First documentation of in vivo and in vitro ivermectin resistance in Sarcoptes scabiei. Clin Infect Dis 2004;39:e8-12.

25. Pasay C, Arlian L, Morgan M, et al. High-resolution melt analysis for the detection of a mutation associated with permethrin resistance in a population of scabies mites. Med Vet Entomol 2008;22: $82-8$.

26. Mounsey KE, Holt DC, McCarthy J, Currie BJ, Walton SF. Scabies: molecular perspectives and therapeutic implications in the face of emerging drug resistance. Future Microbiol 2008;3:57-66. 